\title{
OBJETIVIDAD CONTEXTUAL Y ROBUSTEZ
}

\author{
Sergio F. Martínez
}

Lnstituro de Investigaciones Filosóficas

UNAM

La noción de objetividad tradicional descansa en la distinción entre propiedades primarias y secundarias. Las propiedades primarias, según esta tradición, son las propiedades que los componentes últimos de la realidad tienen intrínsecamente y son los referentes de las leyes de la naturaleza. Las propiedades secundarias son construcciones mentales. Éste es el núcleo de la doctrina del "materialismo" que Hilary Putnam ha criticado en una serie de trabajos. Como parte central de su propuesta dirigida a superar las dificultades de la concepción tradicional Putnam considera que el concepto de objeto es un concepto puramente lógico. Los objetos para Putnam están determinados por la (interpretación de) estructura lógica de las teorías o esquemas descriptivos.

En este trabajo quiero dirigir la atención del lector a una concepción diferente de la objetividad que ha sido importante fuera de la física y que es, cada vez con mayor insistencia, un componente importante en el diseño de teorías en la biología y las ciencias sociales. Según esta concepción la objetividad es una cuestión de grados y de contextos. Pero si la objetividad es una cuestión de grados y de contextos lo mismo debería poder decirse de los objetos de un sistema descriptivo. La existencia y la objetividad deberían verse como una cuestión de grados, así la relatividad conceptual sería una manera de expresar esta idea central.

Empiezo en la segunda sección dando una breve caracterización de la distinción entre propiedades primarias y secundarias y de la noción de objetividad que va asociada con ella. Esto me permitirá hacer ver un supuesto crucial de la tradición. A grandes rasgos, el supuesto es que las propiedades primarias son las propiedades que el objeto tiene independientemente de la existencia de otros existentes ( $y$ más en general independientemente del contexto). En la sección tres muestro con un ejemplo que la distinción tradicional entre propiedades primarias y secundarias es demasiado simplista. La propiedad de tener un cierto largo, cuando examinada con cierto detenimiento, no es una propiedad primaria, ni tampoco puede decirse que es una propiedad secundaria. 
Hay una referencia ineludible a un contexto en cualquier conceptualización del largo (de un objeto) que rebasa la distinción tradicional entre propiedades primarias y secundarias. En la sección cuatro examino brevemente la idea alternativa de la objetividad como robustez. Encontraremos aquí una elaboración pragmática de la idea de que la objetividad es dependiente del contexto. En la sección final muestro que el sentido en el que la objetividad es dependiente de conceptos es en un sentido importante, diferente del tipo de dependencia asociado al realismo interno de Putnam.

\section{II}

La concepción tradicional de la objetividad se origina en el siglo XVII. La idea central de esta concepción, la distinción entre dos tipos de propiedades como base de un examen de la objetividad, no es algo nuevo. Lo que es nuevo es la manera en la que la distinción se hace y se justifica. La lista de las propiedades "primarias" (o "esenciales", "originales" o "simples") dadas por Galileo, Boyle, Newton y Locke difieren en ciertos aspectos pero tienen un núcleo común. ${ }^{1}$ Para Galileo una distinción importante debe hacerse entre las propiedades que no pueden pensarse separadamente de los cuerpos y las propiedades "que uno no se siente obligado a imponer como acompañamiento necesario" (las propiedades que según Galileo "sólo residen en la conciencia"). Galileo utiliza la distinción para argumentar que los cometas no son reales y que el calor no es una sustancia.

Newton piensa que las propiedades primarias son aquellas propiedades que podemos percibir en todos los cuerpos sensibles, y que son aditivos, como diremos utilizando terminología moderna, en el sentido que la propiedad del todo es el resultado de la adición de propiedades de las partes: "las partes más pequeñas de los cuerpos [son...] extendidas, y duras, e impenetrables, y movibles, y con su propia vires inertia. Y esto es el fundamento de toda filosofia. ${ }^{2}$ Newton cree que estas propiedades primarias (lo que llama "las cualidades esenciales" en algunos contextos) pueden caracterizarse físicamente, como las propiedades que entran en la descripción de las leyes fundamentales de la naturaleza. Newton aparentemente piensa que la suposición de la existencia de

1 Galileo nos dice lo siguiente: "Digo ahora que sin importar cómo concibo cualquier sustancia material o corpórea, siento la necesidad de pensar que está limitada en el espacio, que tiene una cierta forma; que es grande o pequeña en relación con otras cosas, que está en cierto lugar específico en un tiempo dado, que está en movimiento o en reposo; que toca o no toca otro cuerpo; que es una en número, o algunas, o muchas." The Assayer, p. 274.

2 Véase la regla III del razonamiento en el libro III de Mathematical Principles or Natural Philosophy and bis System of the World. 
propiedades primarias es la suposición metafísica básica requerida para hacer física. ${ }^{3}$

Según esta concepción tradicional de la base de la objetividad, los objetos reales se distinguen de las ilusiones ("de nada más que los nombres cuando se separan de los seres vivos", como nos dice Galileo en The Assayer), porque los objetos realmente existentes tienen propiedades primarias. Esta concepción supone no sólo que las propiedades primarias son las propiedades que un objeto tiene independientemente de la mente, sino también que son las propiedades que un objeto tiene independientemente de la existencia de cualquier otra cosa en el mundo. Esta idea está implícita en la caracterización de Locke del sustrato material de los objetos (caracterizado por las propiedades primarias). Newton lo formula explícitamente en el contexto de discusiones acerca de las cualidades físicas de los cuerpos. ${ }^{4}$ La caracterización de Newton de las propiedades primarias como ejemplificable por sistemas que pueden existir en el espacio vacío excluye la posibilidad de que una propiedad primaria sea una propiedad relacional de una cierta configuración de partículas. Mas en general excluye la posibilidad de que una propiedad de un cuerpo en un contexto físico no sea reducible (o no sea superveniente) a propiedades físicas de los objetos elementales (caracterizados por sus propiedades primarias).

En lugar de adentrarnos más allá en las sutilezas de la historia del concepto de propiedad primaria simplemente resumo la idea tradicional como sigue: i) las propiedades primarias son propiedades intrínsecas u objetivas que son el caso independientemente del contexto; ii) las propiedades primarias son aditivas (en el sentido mencionado anteriormente); iii) una propiedad primaria no puede explicarse en términos de la existencia de otras propiedades.

La caracterización de las propiedades secundarias siempre ha sido más problemática y controversial. Para nuestro propósito presente es suficiente decir

3 Hay diferencias importantes en las ideas de Newton por un lado y las de Descartes, Boyle y Locke por el otro. Para Newton la metafísica debe de subordinarse a la física y en particular el conjunto de propiedades primarias debe derivarse de los fenómenos. Mcguire 1967 es un examen pertinente de la noción de cualidad fisica en Newton.

4 Newton sugería caracterizar las propiedades primarias de los cuerpos como aquellas propiedades a las que se refieren las leyes fundamentales de la naturaleza (las leyes de la mecánica y la gravitación). Es por esto que Newton considera necesario agregar a la lista de propiedades primarias la inercia de los cuerpos. Pero como Leibniz le objetó, ‘por qué no se incluye también la gravitación? Esto forma parte de la acusación de Leibniz a Newton de que Newton estaba tratando con "propiedades ocultas". La respuesta de Newton puede reconstruirse como sigue. La gravitación es sólo una propiedad interaccional que no se predica de cuerpos aislados, sino sólo de pares de sistemas. Por el contrario, la inercia, como las otras propiedades primarias, es una propiedad que un sistema ejemplificaría aun en el espacio vacío. Así, Newton llega a través de consideraciones físicas a una formulación explícita de la idea de que las propiedades primarias son intrínsecas, en el sentido de que son el caso, independientemente de qué es lo que existe en el mundo además de los portadores de las propiedades primarias.

Recientemente Lewis (1983) ha criticado una versión formulada por Chisholm de esta idea. 
que las propiedades secundarias han sido entendidas como contextuales, no aditivas, y reducibles (en uno u otro sentido) a propiedades primarias.

\section{III}

Tomemos el caso de un ejemplo paradigmático de una propiedad primaria, la propiedad de tener un cierto largo. La concepción tradicional puede fácilmente acomodar el hecho de que en diferentes situaciones fisicas el largo de una mesa puede tener diferentes valores. Ésta es una relatividad inofensiva. Las diferentes situaciones físicas que llevan a la expansión o a la deformación de la mesa, por ejemplo, son independientes de conceptos. Podemos explicar los cambios causalmente. Es claro también que limitaciones obvias en los medios del conocimiento pueden ser aceptadas por esta concepción. Alguien que sostiene la distinción tradicional puede estar de acuerdo en que no hay una medición única privilegiada que nos va a dar el largo de la mesa. Pero se asume que una serie de mediciones nos pueden aproximar cada vez más al largo objetivo de la mesa.

Sin embargo, implícito en este razonamiento hay una suposición importante. Se supone que la mesa es un objeto euclideano y que en particular la superficie medida es plana. Pero que la mesa tenga una superficie plana es una idealización hasta cierto punto arbitraria. Si creemos lo que vemos a través del microscopio la superficie es bastante irregular. Si medimos el largo de la mesa por medio de los pasos de un pequeño robot microscópico (digamos que del tamaño de una molécula de agua) que camine a lo largo de la superficie de la mesa, el resultado de esta medición sería (muy probablemente) diferente a la medición estándar. Y si medimos el largo de la mesa con un robot más pequeño, el resultado sería (muy probablemente) diferente a los dos resultados anteriores. $^{5}$

Esto puede resumirse diciendo que no hay una propiedad de largo independientemente del grano del detector (i.e. independientemente de la unidad de medición y del proceso específico de medición que se escojan). Implícitamente el procedimiento estándar asume que la mesa es un objeto cuclideano. Pero esta mesa puede modelarse como un objeto euclideano o como un objeto no euclideano (fractal); la mesa no tiene una etiqueta que diga que es tal o cual tipo de objeto geométrico. Nosotros ponemos las etiquetas. La razón

$5 \mathrm{Si}$ los resultados coinciden o no (o si convergen o divergen) depende de la mesa (y del proceso de medición) en particular. Este ejemplo es una modificación del famoso ejemplo de Mandelbrot acerca del largo de la costa de la Gran Bretaña (véase Mandelbrot, 1983). Como Mandelbrot muestra, la medida del "largo" que obtenemos como resultado de los diferentes métodos de medición (los pasos de pequeños robots en nuestro ejemplo) pueden diverger o converger a diferentes valores. 
de por qué hay acuerdo intersubjetivo acerca de la idealización de la mesa como un objeto euclideano es que nuestro aparato sensorial tiene un "grano" intrínseco que nos hace ver la mesa como un objeto plano euclideano (y que nos hace percibir la mesa como lisa al tacto).

Sobre la base de las consideraciones anteriores, si queremos seguir hablando de "la propiedad de tener un cierto largo", asumiendo implícitamente que estamos hablando del largo módulo de nuestro aparato detector (nuestro aparato sensorial), entonces deberíamos pensar que la propiedad de "tener un cierto largo" es una propiedad dependiente de la mente; depende de la manera en la que las cosas externas nos afectan. La propiedad de "tener un cierto largo" es una propiedad relacional que se da entre nuestro aparato sensorial y la cosa externa. Pero si queremos hablar de "la propiedad de un cierto largo módulo del grano del detector", entonces el largo no sería una propiedad dependiente de la mente. Su caracterización no requiere un elemento subjetivo, una afección de la mente, pero es una propiedad dependiente del contexto. A grandes rasgos, el contexto está dado por el grano del detector.

Hay dos razones para detenernos en este ejemplo. En primer lugar, nótese que la dependencia conceptual en este caso no significa dependencia a una teoría específica, o dependencia a un "sentido común". Esto sugiere que la manera en la que los conceptos se relacionan con objetos es muy compleja y no es susceptible de generalización filosofica sin recursos pragmáticos adicionales como el de contextualización. Segundo, muestra que el modelo tradicional de la objetividad basado en la distinción entre propiedades primarias y secundarias no puede mantenerse. Sin embargo, el ejemplo también sugiere un posible remedio. Una vez que nos damos cuenta que el atribuir una propiedad tan básica como el largo a un cuerpo físico involucra una idealización, entonces el problema de si la propiedad es primaria o secundaria no puede ser realmente un problema. Lo importante es encontrar los conceptos que discriminan entre los diferentes contextos en los que las propiedades pueden definirse como independientes de la mente. 6

\section{IV}

El intento por distinguir diferentes tipos de propiedades como el punto de partida para una distinción entre la ilusión y la realidad (y por lo tanto como una caracterización de la objetividad) es muy antiguo. Aristóteles en $D e A n i-$ ma menciona algunos intentos en esta dirección debatidos en tiempos presocráticos y elabora su propia versión de esta distinción. Aristóteles piensa que

6 Por lo general estos conceptos provienen de teorías, pero esto no es necesario. Un buen ejemplo de conceptualización no proveniente de teorias son los conceptos propuestos por Torretti en 1990 (en la sección 4.7) para entender la noción de azar en los casos en los que no podemos basar esta noción de azar en la existencia de condiciones experimentales definidas de azar (definite chance set-up). 
"debemos hablar en primer lugar de los objetos de la percepción en relación con cada uno de los sentidos" (De Anima, 418a7). Cada uno de los sentidos tiene sus "objetos especiales" que pueden no ser percibidos por los otros sentidos. Pero hay también "objetos del sentido común" que son perceptibles por más de un sentido. Aristóteles menciona el movimiento, el reposo, el número, la figura y el tamaño como (propiedades de) objetos del sentido común. Aristóteles sugiere que los objetos del sentido común tienen una mayor posibilidad de captar nuestra atención y una menor probabilidad de que nos engañen. Viendo un líquido amarillo podemos pensar que es miel o bilis. Si es sólo como objeto especial (como objeto visual por ejemplo) que lo percibimos, muy probablemente no podríamos decir si es lo uno o lo otro. Si tenemos acceso al tacto (o al gusto) nos permitiría casi seguro discriminar entre los dos tipos de objeto.

Este criterio de la objetividad en términos de la "robustez" sensorial está en el centro de una abigarrada tradición en el renacimiento italiano. Uno de los temas centrales de la teoría de la mente de Ficino, su teoría de los fantasmas, es que hay dos tipos diferentes de fantasmas. Algunos fantasmas son por lo menos ilusiones parciales, puesto que son engendros de un único tipo de sensación. Los fantasmas más legítimos no son sólo audiovisuales o auditivos, sino que surgen de la colaboración de varios o todos los sentidos simultáneamente.

Más recientemente, Donald Campbell ha sugerido que la característica de nuestros objetos del sentido común es que pueden "diagnosticarse" de diferentes maneras, y que algo es ilusorio en la medida en la que los límites derivados de una modalidad sensorial no se refuerzan como es de esperarse con los límites correlativos de otras modalidades sensoriales. Él ha generalizado esta idea en su método del "Operacionalismo múltiple" (véase Campbell, 1988a y 1988b). Otras generalizaciones del criterio de robustez sensorial han sido elaborados en una serie de teorías en psicología y las ciencias sociales por medio de la incorporación de la noción de "robustez estadística". 7 Esta noción de objetividad como robustez es muy importante en la construcción y en la evaluación de modelos en las ciencias empíricas.

En el sentido en el que quiero centrarme en este trabajo, los modelos son representaciones idealizadas de los fenómenos. Es común pensar los modelos como modelos-de-teorías. Por ejemplo, cuando Newton trata de reducir la óptica a la mecánica por medio de la construcción de un modelo mecánico del comportamiento de la luz, él está tratando de hacer un modelo mecánico de la óptica en este sentido. Quiero centrarme aquí en el uso de los modelos como herramientas para la detección y la sistematización de fenómenos, y

7 En Wimsatt, 1980 y 1981 hay un examen de los diferentes sentidos de robustez y de sus aplicaciones a cuestiones en la filosofía de la ciencia. 
tratar de mantenerme en lo posible alejado de los modelos como modelos de teorías.

Los mapas pueden ser un ejemplo paradigmático de modelos en el sentido no teórico que aquí queremos recalcar. Si estamos tratando de entender un mapa no tiene mucho sentido usar un lente de aumento para interpretar las manchas amarillas irregulares que pueden estar en el papel. Como tampoco nos ayudaría a orientarnos el tratar de intepretar las irregularidades en la textura del papel del mapa como correspondiente a las irregularidades del terreno. Una razón importante detrás de esta seguridad en lo que podemos dejar fuera de consideración en un mapa es la manera como estamos hechos biológicamente. Al igual que en el caso del ejemplo de la mesa que examinamos en la sección tres, los mapas vienen con propiedades contextuales que son discernibles sobre la base de convenciones y acuerdos intersubjetivos. Este acuerdo presupone un aparato sensorial similarmente diseñado por la evolución. ${ }^{8}$

Sin embargo, esta característica de los mapas, la distinción (relativamente) clara entre la información que se desea transmitir y el "fondo", no es una propiedad de una serie de modelos utilizados en las ciencias empíricas. En la biología de poblaciones encontramos una amplia variedad de ejemplos. Como Levins nos dice (1966, p. 423):

There is always room for doubt as to whether a result depends on the essentials of a model, or on the details of the simplifying assumptions. This problem does not arise in the more familiar models, such as the geographical map, where we all know that contiguity on the map implies contiguity in reality, relative distances on the map correspond to relative distances in reality, but color is arbitrary and a microscopic view of the map would only show the fibers on the paper on which is printed. But in the mathematical models of population biology, it is not always obvious when we are using too high a magnification.

Nótese que las propiedades "arbitrarias" mencionadas por Levins son ejemplos típicos de propiedades secundarias y que las propiedades que "todos sabemos cómo interpretar" son ejemplos típicos de propiedades primarias (véase la cita de Galileo en la nota 1). Esto sugiere que las propiedades primarias tradicionales tienen una cierta "robustez" que las hace útiles como portadoras de contenido semántico en mapas "de sentido común", como mapas. El acuerdo entre Galileo y Aristóteles y muchos filósofos contemporáneos acerca de lo que es una propiedad primaria no surge simplemente de compartir una

8 Si alguno de nosotros tuviera visión telescópica y otros visión microscópica con diferentes aumentos, o si hubiera grupos de seres humanos que usaran diferentes propiedades de la luz para ver, entonces los mapas no serían modelos simples. Tendríamos que desarrollar un sistema altamente sofisticado de convenciones y símbolos para que los mapas cumplieran la función que ahora cumplen sin mucho esfuerzo de nuestra parte. 
tradición metafísica, surge en parte del hecho de compartir un sentido común que fuertemente sugiere la concepción tradicional.

Levins, en el párrafo que sigue a la cita anterior, nos dice que en la biología de poblaciones es necesario introducir un criterio de robustez para distinguir lo que está allí objetivamente y lo que es un mero artefacto de nuestros modelos. Levins formula esta idea como sigue:

[When] several alternative models, each with different simplifications, but with a common biological assumption [...] lead to similar results we have what we can call a robust theorem that is relatively free of the details of the model. Hence our truth is the intersection of independent lies. (1966, p. 423)

Este criterio de robustez de Levins (que ha sido posteriormente adaptado por Wimsatt para el replanteamiento de una serie de cuestiones filosóficas) es una generalización del criterio sugerido por Aristóteles, Ficino y Campbell. En lugar de las diferentes modalidades sensoriales la biología de poblaciones busca una variedad de modelos independientes.

\section{V}

Según esta concepción de la objetividad como derivada de la robustez, la objetividad es contextual y una cuestión de grados. Sin embargo, como en el ejemplo examinado en la sección tres y en la situación descrita en la biología de poblaciones por Levins, el sentido en el que la objetividad es contextual no parece tener mucho que ver con el tipo de contextos de los que habla el realismo interno de Putnam. Según Putnam los objetos son algo interno de los esquemas conceptuales (o lenguajes o esquemas descriptivos). Pero en los ejemplos examinados arriba y en general según la concepción de objetividad como robustez, los objetos surgen por asi decir de la concordancia de las diferentes descripciones.

La noción de independencia es crucial para esta concepción de objetividad. Es la existencia de descripciones independientes lo que cuenta para decidir la manera en la que los objetos se recortan (véase Campbell, 1988b; Wimsatt, 1981). En el realismo interno no existe una noción similar o análoga a la de independencia que es parte integral de nuestra caracterización de lo que es un objeto.

La fundamentación de la epistemología en la distinción entre propiedades primarias y secundarias supone que la objetividad es una cuestión de todo o nada. El realismo interno de Putnam conserva un rasgo distintivo de esta idea. Supone que la relativización de las propiedades primarias sólo puede darse por medio de su reinterpretación como propiedades secundarias. Pero que las propiedades que caracterizan lo objetivo sean contextuales no tiene 
por qué entenderse como la imposibilidad de separar el aspecto objetivo de lo "proyectado" por nosotros.

La contextualidad y la independencia no son nociones precisas. Tienen que hacerse precisas caso por caso. El sentido en el que el largo ha sido caracterizado como una propiedad contextual en la sección tres no es el mismo sentido en el que un teorema robusto es dependiente del contexto. Pero hay importantes similaridades en las nociones de contexto $\mathrm{e}$ independencia que parecen estar en el núcleo de nuestra noción de objetividad en ciencia y en el sentido común.

Si pensamos en los objetos como las proyecciones robustas de nuestras descripciones, el esquema del sentido común y el esquema de la ciencia no tienen por qué verse como en conflicto (como lo sugiere Putnam). Al contrario, deben verse como si se apoyaran mutuamente. Y ésta parece una característica general de la manera en la que nuestros conceptos entran en la construcción de la realidad.

\section{Bibliografía}

Campbell, D., 1988, Methodology and Epistemology for Social Science, Selected papers, The U. of Chicago Press, Chicago.

Galileo, The Assayer, en Discoveries and Opinions of Galileo (1623), trad., introd. y notas de Stillman Drake, 1957, pp. 229-280.

Levins, R., 1966, "The Strategy of Model Building in Popularion Biology", American Scientist, no. 54, pp. 421-431.

Lewis, D., 1983, “Extrinsic Properties”, Philosophical Studies, no. 44, pp. 197-200.

Mandelbrot, B., 1983, The Fractal Geometry of Nature, ed. actualizada y aumentada, W.H. Freeman, Nueva York.

Martínez, S., 1992, "Internal Versus Contextual Reality: the Case of Quantum Mechanics", ms.

Mcguire, P., 1967, "Transmutation and Immutability: Newton's Doctrine of Physical Qualities", Ambix, vol. XIV, junio.

Newton, I., Mathematical Principles or Natural Philosophy and his System of the World (1686), trad. de A. Motte en 1729, revisado por F. Cajori, reedición de U. of California Press en 1934, 6a. impresión, 1966.

Putnam, H., 1983, Realism and Reason. Philosophical Papers III, Cambridge U. Press.

—, 1987, The Many Faces of Realism, Open Court, LaSalle (III.).

—, 1990, Realism with a Human Face, comp. e introd. de J. Conant, Harvard U. Press.

Torretti, R., 1990, Creative Understanding, Philosophical Reflections on Physics, The University of Chicago Press. 
Wimsatt, 1981, "Robustness, Reliability and Overdetermination" en M.B. Brewer y B.E. Collins (comps.), Scientific Inquiry and the Social Sciences (volumen en honor a Donald T. Campbell), Jossey-Bass, San Francisco, pp. 124-163.

, 1980, "Ramdomness and Perceived Ramdomness in Evolutionary Biology", Synthese, no. 43, pp. 287-329. 